\title{
Análisis de los factores que determinan el diseño de mallas metálicas para la estabilización de taludes en macizos rocosos
}

\author{
Analysis of the factors that determine the wire mesh design for the stabilization of rock slopes
}

Fecha de entrega: 30 de enero de 2017

Fecha de aceptación: 24 de mayo 2018

\section{Karen Lizeth Grattz, Juan Diego Salazar y Carlos E. Rodríguez}

\begin{abstract}
Departamento de Ingeniería Civil, Pontificia Universidad Javeriana, Carrera 7 N 40-62, Bogotá D.C., Colombia, kgrattz@javeriana.edu.co,jsalazarh@javeriana.edu.co,crodriguezp@javeriana.edu.co
\end{abstract}

Las caidas de rocas son un tipo de inestabilidad usual y frecuente en taludes en roca, la necesidad de protección frente a este tipo de fenómenos ha llevado al desarrollo de diferentes soluciones, entre ellas encontramos la estabilización de taludes mediante el uso de sistemas de malla metálica con pernos o anclajes. Los fabricantes han desarrollado manuales y softwares de diseño de estas mallas, sin embargo, estas herramientas son de uso exclusivo y con fines comerciales. Así mismo el desconocimiento general del diseño e implantación de estas soluciones, relacionado a la falta de claridad en el funcionamiento de estos elementos sobre el macizo rocoso, restringe el diseño y la colocación de este tipo de soluciones únicamente a los fabricantes. Teniendo en cuenta lo anterior, el presente trabajo tiene como objetivo establecer la influencia de las propiedades mecánicas y geométricas de los macizos rocosos y mecánicas de las mallas flexibles en el diseño de sistemas de estabilización para taludes en macizos rocosos, para lo cual se propone una nueva metodología para el diseño de estabilizaciones de taludes en macizos rocosos con mallas metálicas flexibles.

Palabras clave: estabilidad de taludes, malla metálica, roca, perno
Rock falls are a type of instability that is frequent in rock slopes. The need for protection against these types of phenomena has led to the development of different solutions, including stabilization of slopes with the use of wire mesh with bolts or anchors. The manufacturers have developed manuals and design software for these meshes, however, these tools are for commercial purposes. Likewise, the general lack of knowledge about the design and implementation of these solutions, restricts the design and placement of systems to the manufacturers. The present work aims to establish the influence of the mechanical and geometrical properties of the mass of rock and mechanical of the meshes in the design of stabilization systems for slopes, for which a new design methodology is proposed.

Keywords: slope stability, metallic mesh, rock, nail

\section{Introducción}

El sistema de transporte carretero es uno de los modos de movilización de pasajeros y mercancías más importantes a nivel mundial debido a su amplia flexibilidad. Dentro del total de movilización de carga en Colombia el modo carretero es el más importante con un $73.2 \%$ del total de la carga transportada. En este orden de ideas, ha surgido la necesidad de desarrollar soluciones de ingeniería con miras a brindar corredores seguros y eficientes a los usuarios. Particularmente en el área Geotécnica en Colombia, cada día es más evidente la vulnerabilidad en la que se encuentra sometida la infraestructura vial, y las poblaciones por los deslizamientos, que se presentan principalmente por dos factores detonantes: las lluvias y los sismos (Ramos et al., 2015). De ahí que los principales problemas asociados a riesgos geológicos propios de las carreteras de montaña, la topografía de las zonas donde se generan gran cantidad de estos proyectos viales, la geología joven y alta sismicidad a la que estarían expuestos los taludes, generan escenarios propicios para las caídas de bloques de roca de los macizos.

Las caídas de rocas son un tipo de inestabilidad usual en laderas escarpadas y montañosas o taludes cortados en roca (Fariñas de Alba et al., 2008). Un ejemplo de esta situación es la presentada por Salazar Hernández (2012), quien identificó que, tan solo el sismo de Quetame de 2008, 
tuvo efectos cosísmicos en la generación de 68 procesos de remoción en masa, donde más de $50 \%$ correspondió a caídas de rocas. Al menos dos de estos procesos se presentaron en la vía Bogotá-Villavicencio, Colombia, causando cuantiosas pérdidas económicas debido al alto tráfico promedio diario y a la importancia de esta vía para el transporte de productos agrícolas e hidrocarburos en la época.

A nivel internacional, se ha documentado la recurrencia de deslizamientos durante eventos sísmicos, dando lugar al desarrolló de bases de datos de deslizamientos inducidos por sismos. Caballero Chaves (2011) reportó, el análisis de frecuencia por tipo de mecanismo, donde situó la caída de rocas como el tercer mecanismo de mayor recurrencia, con un $12.9 \%$ de los mecanismos muestreados. La necesidad de generar protección ante estos procesos, ha llevado al desarrollo de diferentes tipos de soluciones tecnológicas de ingeniería. Estas soluciones previenen la ruptura de los bloques desde la pared del macizo o ayudan a controlar, interceptar o desviar los bloques durante el movimiento. Los dispositivos más recientes incluyen zanjas, terraplenes de suelos y cercas hechas de mallas metálicas (Peila et al., 2007).

Las soluciones denominadas sistemas flexibles, constituyen una técnica para la estabilización superficial de los taludes, compuesta principalmente por membranas de mallas, cables y pernos anclados al terreno. La técnica es ampliamente extendida debido al bajo impacto visual y su mínima influencia sobre el tráfico durante su instalación (Blanco-Fernandez et al., 2011). Si bien existen algunas metodologías para el diseño de estas soluciones, la mayor parte se han basado principalmente en criterios empíricos (Blanco-Fernandez et al., 2011). También se ha utilizado el software Flac3D para realizar análisis de la influencia del empleo de las membranas de acero en el incremento de la estabilidad de sistemas de anclajes empleando modelos de cuña, deslizamiento planar y volcamiento (Cardoso, 2005). Sin embargo, el uso de este software es complejo y no está fácilmente disponible para el diseño regular de soluciones de estabilización.

Los fabricantes han desarrollado manuales y softwares de diseño de estas mallas (e.g. Cała et al., 2012), sin embargo, estas herramientas son de uso exclusivo y con fines comerciales. Así mismo el desconocimiento general del diseño e implantación de estas soluciones, relacionado a la falta de claridad en el funcionamiento de estos elementos sobre el macizo rocoso, restringe el diseño y la colocación de este tipo de soluciones únicamente a los fabricantes.

Teniendo en cuenta lo anterior, el presente trabajo tiene como objetivo establecer la influencia de las propiedades mecánicas y geométricas de los macizos rocosos y mecánicas de las mallas flexibles en el diseño de sistemas de estabilización para taludes en macizos rocosos, para lo cual se propone una nueva metodología para el diseño de estabilizaciones de taludes en macizos rocosos con mallas metálicas flexibles.

\section{Metodología para el cálculo de estabilidad de taludes en roca}

Para desarrollar la siguiente metodología de cálculo del factor se seguridad para un talud en estado natural y estabilizado con la malla, se tomaron como base las ecuaciones de estabilidad planteadas por Hoek y Bray (2014) en los capítulos 7, 8, 10 y apéndice 2. Igualmente se toma como hipótesis de diseño que el sistema de estabilización trabaja de manera activa ejerciendo una presión sobre el talud desde el momento de su instalación.

\section{Falla planar}

\section{Análisis estático}

La Figura 1 muestra el talud y la superficie de falla del problema a resolver. El factor de seguridad está dado por la relación entre las fuerzas desestabilizantes y las fuerzas resistentes:

$$
\begin{aligned}
& F S=\frac{\text { Fuerzas resistentes }}{\text { Fuerzas desestabilizantes }} \\
& F S=\frac{c A+\left(W \cos \phi_{p}-U-V \operatorname{sen} \phi_{p}\right) \tan \phi}{W \operatorname{sen} \phi_{p}+V \cos \phi_{p}}
\end{aligned}
$$

donde $A$ es el área del plano de falla, $c$ y $\phi$ son la cohesión y el ángulo de fricción en el plano de falla, $U$ es la fuerza de empuje del agua sobre el plano de falla, $V$ es la fuerza de empuje del agua en la grieta de tracción y $W$ es el peso del bloque que se desliza.

El área del plano de falla se puede expresar como sigue:

$$
\begin{aligned}
& A=L \cdot 1 \\
& L=h_{2} \csc \phi_{p}
\end{aligned}
$$




$$
\begin{aligned}
& h_{2}=H+b \tan \phi_{s}-Z \\
& L=\left(H+b \tan \phi_{s}-Z\right) \csc \phi_{p} \\
& A=\left[\left(H+b \tan \phi_{s}-Z\right) \csc \phi_{p}\right] \cdot 1
\end{aligned}
$$

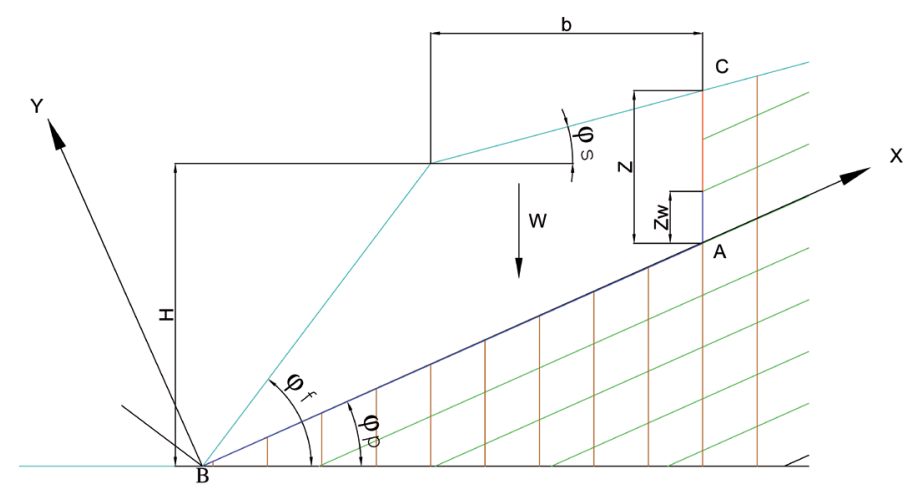

Figura 1: Geometría para cálculo por falla planar

La fuerza de empuje $U$ que ejerce el agua sobre el plano de falla está dada por el gradiente hidráulico a lo largo de la misma, donde $\gamma_{\mathrm{w}}$ es el peso unitario del agua. Se asumirá que el gradiente hidráulico varía de forma lineal a lo largo del plano de falla por lo tanto se puede calcular la presión desestabilizante ejercida considerando la altura de presión en el punto A, $h_{\mathrm{a}}, \mathrm{B}, h_{\mathrm{b}}$ y C, $h_{\mathrm{c}}$, de la siguiente manera:

$$
\begin{aligned}
& U=\frac{1}{2}\left(h_{a}+h_{b}\right) \gamma_{w} L \\
& U=\frac{1}{2}\left(h_{a}+h_{b}\right) \gamma_{w}\left[\left(H+b \tan \phi_{s}-Z\right) \csc \phi_{p}\right]
\end{aligned}
$$

La fuerza de empuje del agua $V$ en la grieta de tracción.

$$
V=\frac{1}{2}\left(h_{a}+h_{c}\right) \gamma_{w} Z
$$

El peso del bloque $W$ que desliza para un peso unitario $\gamma$ de la roca, se puede calcular de la siguiente manera:

$$
W=\gamma\left(\frac{1}{2}\left[b \tan \phi_{s}\right] b+\frac{1}{2} \frac{H^{2}}{\tan \phi_{f}}+b H-\frac{1}{2} h_{2} L \cos \phi_{p}\right) \cdot 1
$$

Si se considera una fuerza distribuida $P$ ejercida por la malla sobre la superficie del talud como se muestra en la Figura 2, el factor de seguridad FS se calcula de la siguiente manera

$$
F S=\frac{c A+\left[W \cos \phi_{p}-U-V \operatorname{sen} \phi_{p}+P \frac{H}{\cos \left(90-\phi_{f}\right)} \cos \left(\phi_{f}-\phi_{p}\right)\right] \tan \phi}{W \operatorname{sen} \phi_{p}+V \cos \phi_{p}-P \frac{H}{\cos \left(90-\phi_{f}\right)} \operatorname{sen}\left(\phi_{f}-\phi_{p}\right)}
$$

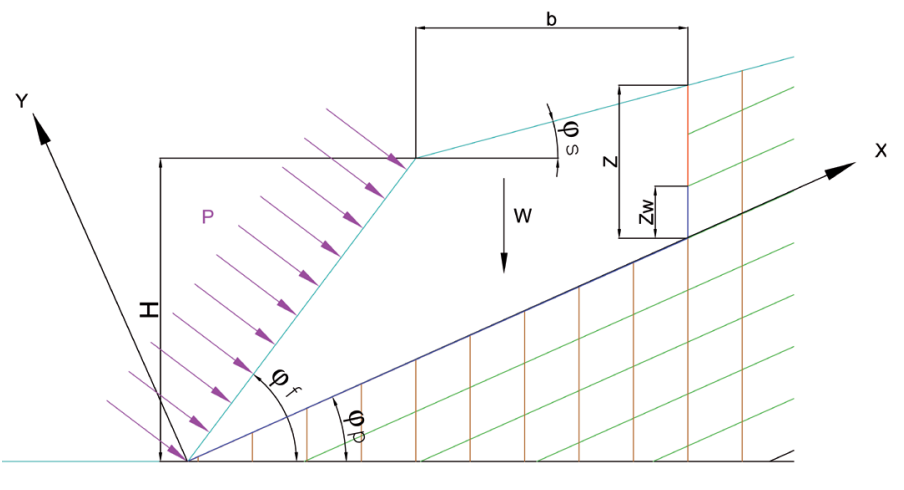

Figura 2: Fuerza distribuida ejercida por la malla

\section{Análisis pseudo-estático}

El procedimiento de análisis pseudo-estático simula los movimientos del suelo como una fuerza estática que actúa desestabilizando el talud. La magnitud de esta fuerza está dada por el producto entre el peso del bloque deslizante $W$ y un coeficiente sísmico $k$ que actúa en dirección $\phi_{\mathrm{k}}$ El Factor de seguridad para el caso pseudo-estático teniendo en cuenta la fuerza sísmica se calcula de la siguiente manera:

$$
F S=\frac{c A+\left(W \cos \phi_{p}-U-V \operatorname{sen} \phi_{p}-W k\left(\operatorname{sen} \phi_{p}+\phi_{k}\right)\right) \tan \phi}{W \operatorname{sen} \phi_{p}+V \cos \phi_{p}+W k\left(\cos \phi_{p}+\phi_{k}\right)}
$$

Para los casos en los cuales se requiera la utilización de malla metálica para estabilización del talud, el factor de seguridad se calculará de la siguiente manera:

$F S=\frac{c A+\left(W \cos \phi_{p}-U-V \operatorname{sen} \phi_{p}-W k\left(\operatorname{sen} \phi_{p}+\phi_{k}\right)+P \frac{H}{\cos \left(90-\phi_{f}\right)} \cos \left(\phi_{f}-\phi_{p}\right)\right) \tan \phi}{W \operatorname{sen} \phi_{p}+V \cos \phi_{p}+W k\left(\cos \phi_{p}+\phi_{k}\right)-P \frac{H}{\cos \left(90-\phi_{f}\right)} \operatorname{sen}\left(\phi_{f}-\phi_{p}\right)}$

\section{Falla en cuña}

\section{Análisis estático}

La Figura 3 muestra una vista en perspectiva de la cuña de falla, donde los parámetros de entrada son: buzamiento del plano A, $\phi_{a}$, dirección de buzamiento plano A, $\alpha_{a}$, buzamiento plano $\mathrm{B}, \phi_{\mathrm{b}}$, dirección de buzamiento plano $\mathrm{B}$, $\alpha_{b}$, buzamiento cara del talud $\phi_{\mathrm{f}}$, dirección de buzamiento cara del talud $\alpha_{\mathrm{f}}$, buzamiento talud superior $\phi_{\mathrm{s}}$, dirección de buzamiento talud superior $\alpha_{\mathrm{s}}$, buzamiento grieta de tracción $\phi_{\mathrm{t}}$, dirección de buzamiento grieta de tracción $\alpha_{\mathrm{t}}$, distancia AO medida en campo y altura de la grieta de tracción $Z$. 


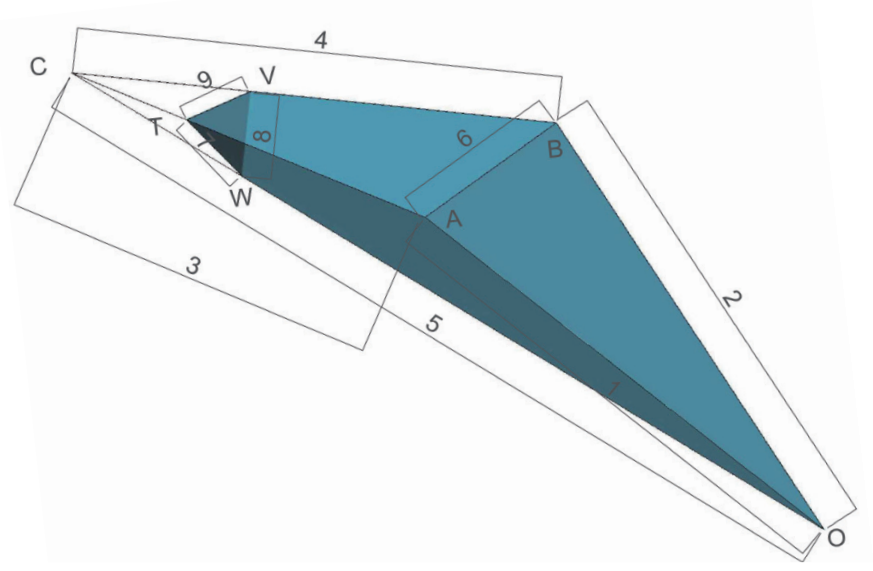

Figura 3: Geometría de la cuña de falla y terminología

Las direcciones de buzamiento de cada una de las líneas que componen la geometría del bloque inestable se calculan de la siguiente manera:

$$
\begin{aligned}
& \alpha_{1}=\arctan \left[\frac{\tan \phi_{a} \cos \alpha_{a}-\tan \phi_{f} \cos \alpha_{f}}{\tan \phi_{f} \operatorname{sen} \alpha_{f}-\tan \phi_{a} \operatorname{sen} \alpha_{a}}\right] \\
& \alpha_{2}=\arctan \left[\frac{\tan \phi_{b} \cos \alpha_{b}-\tan \phi_{f} \cos \alpha_{f}}{\tan \phi_{f} \operatorname{sen} \alpha_{f}-\tan \phi_{b} \operatorname{sen} \alpha_{b}}\right] \\
& \alpha_{3}=\arctan \left[\frac{\tan \phi_{a} \cos \alpha_{a}-\tan \phi_{s} \cos \alpha_{s}}{\tan \phi_{s} \operatorname{sen} \alpha_{s}-\tan \phi_{a} \operatorname{sen} \alpha_{a}}\right] \\
& \alpha_{4}=\arctan \left[\frac{\tan \phi_{b} \cos \alpha_{b}-\tan \phi_{s} \cos \alpha_{s}}{\tan \phi_{s} \operatorname{sen} \alpha_{s}-\tan \phi_{b} \operatorname{sen} \alpha_{b}}\right] \\
& \alpha_{5}=\arctan \left[\frac{\tan \phi_{a} \cos \alpha_{a}-\tan \phi_{b} \cos \alpha_{b}}{\tan \phi_{b} \operatorname{sen} \alpha_{b}-\tan \phi_{a} \operatorname{sen} \alpha_{a}}\right] \\
& \alpha_{6}=\arctan \left[\frac{\tan \phi_{f} \cos \alpha_{f}-\tan \phi_{s} \cos \alpha_{s}}{\tan \phi_{s} \operatorname{sen} \alpha_{s}-\tan \phi_{f} \operatorname{sen} \alpha_{f}}\right] \\
& \alpha_{7}=\arctan \left[\frac{\tan \phi_{a} \cos \alpha_{a}-\tan \phi_{t} \cos \alpha_{t}}{\tan \phi_{t} \operatorname{sen} \alpha_{t}-\tan \phi_{a} \operatorname{sen} \alpha_{a}}\right] \\
& \alpha_{8}=\arctan \left[\frac{\tan \phi_{b} \cos \alpha_{b}-\tan \phi_{t} \cos \alpha_{t}}{\tan \phi_{t} \operatorname{sen} \alpha_{t}-\tan \phi_{b} \operatorname{sen} \alpha_{b}}\right] \\
& \alpha_{9}=\arctan \left[\frac{\tan \phi_{s} \cos \alpha_{s}-\tan \phi_{t} \cos \alpha_{t}}{\tan \phi_{t} \operatorname{sen} \alpha_{t}-\tan \phi_{s} \operatorname{sen} \alpha_{s}}\right]
\end{aligned}
$$

El buzamiento de cada una de las líneas que componen la geometría del bloque inestable se calcula de la siguiente manera:

$$
\begin{aligned}
& \phi_{1}=\arctan \left(\tan \phi_{a} \cos \left(\alpha_{a}-\alpha_{1}\right)\right) \\
& \phi_{2}=\arctan \left(\tan \phi_{b} \cos \left(\alpha_{b}-\alpha_{2}\right)\right) \\
& \phi_{3}=\arctan \left(\tan \phi_{a} \cos \left(\alpha_{a}-\alpha_{3}\right)\right) \\
& \phi_{4}=\arctan \left(\tan \phi_{b} \cos \left(\alpha_{b}-\alpha_{4}\right)\right) \\
& \phi_{5}=\arctan \left(\tan \phi_{a} \cos \left(\alpha_{a}-\alpha_{5}\right)\right) \\
& \phi_{6}=\arctan \left(\tan \phi_{f} \cos \left(\alpha_{f}-\alpha_{6}\right)\right) \\
& \phi_{7}=\arctan \left(\tan \phi_{a} \cos \left(\alpha_{a}-\alpha_{7}\right)\right) \\
& \phi_{8}=\arctan \left(\tan \phi_{b} \cos \left(\alpha_{b}-\alpha_{8}\right)\right) \\
& \phi_{9}=\arctan \left(\tan \phi_{s} \cos \left(\alpha_{s}-\alpha_{9}\right)\right)
\end{aligned}
$$

Con el fin de determinar las fuerzas que actúan en la cuña, es necesario calcular las áreas de los planos sobre los cuales la cuña se desliza y el volumen de la misma. Para realizar este procedimiento es necesario obtener los ángulos que se forman entre las líneas de intersección, los cuales se calculan mediante las siguientes expresiones:

$$
\begin{aligned}
& \theta_{13}=\arccos \left(\cos \phi_{1} \cos \phi_{3} \cos \left(\alpha_{1}-\alpha_{3}\right)+\operatorname{sen} \phi_{1} \operatorname{sen} \phi_{3}\right) \\
& \theta_{15}=\arccos \left(\cos \phi_{1} \cos \phi_{5} \cos \left(\alpha_{1}-\alpha_{5}\right)+\operatorname{sen} \phi_{1} \operatorname{sen} \phi_{5}\right) \\
& \theta_{24}=\arccos \left(\cos \phi_{2} \cos \phi_{4} \cos \left(\alpha_{2}-\alpha_{4}\right)+\operatorname{sen} \phi_{2} \operatorname{sen} \phi_{4}\right) \\
& \theta_{25}=\arccos \left(\cos \phi_{2} \cos \phi_{5} \cos \left(\alpha_{2}-\alpha_{5}\right)+\operatorname{sen} \phi_{2} \operatorname{sen} \phi_{5}\right) \\
& \theta_{34}=\arccos \left(\cos \phi_{3} \cos \phi_{4} \cos \left(\alpha_{3}-\alpha_{4}\right)+\operatorname{sen} \phi_{3} \operatorname{sen} \phi_{4}\right) \\
& \theta_{35}=\arccos \left(\cos \phi_{3} \cos \phi_{5} \cos \left(\alpha_{3}-\alpha_{5}\right)+\operatorname{sen} \phi_{3} \operatorname{sen} \phi_{5}\right) \\
& \theta_{37}=\arccos \left(\cos \phi_{3} \cos \phi_{7} \cos \left(\alpha_{3}-\alpha_{7}\right)+\operatorname{sen} \phi_{3} \operatorname{sen} \phi_{7}\right) \\
& \theta_{45}=\arccos \left(\cos \phi_{4} \cos \phi_{5} \cos \left(\alpha_{4}-\alpha_{5}\right)+\operatorname{sen} \phi_{4} \operatorname{sen} \phi_{5}\right) \\
& \theta_{48}=\arccos \left(\cos \phi_{4} \cos \phi_{8} \cos \left(\alpha_{4}-\alpha_{8}\right)+\operatorname{sen} \phi_{4} \operatorname{sen} \phi_{8}\right) \\
& \theta_{49}=\arccos \left(\cos \phi_{4} \cos \phi_{9} \cos \left(\alpha_{4}-\alpha_{9}\right)+\operatorname{sen} \phi_{4} \operatorname{sen} \phi_{9}\right) \\
& \theta_{57}=\arccos \left(\cos \phi_{5} \cos \phi_{7} \cos \left(\alpha_{5}-\alpha_{7}\right)+\operatorname{sen} \phi_{5} \operatorname{sen} \phi_{7}\right) \\
& \theta_{58}=\arccos \left(\cos \phi_{5} \cos \phi_{8} \cos \left(\alpha_{5}-\alpha_{8}\right)+\operatorname{sen} \phi_{5} \operatorname{sen} \phi_{8}\right) \\
& \theta_{79}=\arccos \left(\cos \phi_{7} \cos \phi_{9} \cos \left(\alpha_{7}-\alpha_{9}\right)+\operatorname{sen} \phi_{7} \operatorname{sen} \phi_{9}\right) \\
& \theta_{89}=\arccos \left(\cos \phi_{8} \cos \phi_{9} \cos \left(\alpha_{8}-\alpha_{9}\right)+\operatorname{sen} \phi_{8} \operatorname{sen} \phi_{9}\right)
\end{aligned}
$$

Una vez que se establecen los buzamientos, las direcciones de buzamiento de las líneas de intersección de los planos que forman la cuña y los ángulos que se forman entre las mismas, se pueden calcular las áreas de todos ellos y el volumen de la cuña teniendo como insumo la longitud de una de las líneas de intersección. Cualquiera de las líneas puede ser usada para definir la geometría del bloque, sin embargo, es conveniente basar los siguientes cálculos en una línea cuya dimensión pueda obtenerse en campo. La longitud de la línea $\mathrm{AO}$, puede ser determinada en campo debido a su accesibilidad. El área del plano A viene dada por:

$$
A_{a}=\frac{1}{2}\left[A C^{2} \frac{\operatorname{sen} \theta_{13}}{\operatorname{sen} \theta_{15}}-T C^{2} \frac{\operatorname{sen} \theta_{37}}{\operatorname{sen} \theta_{57}}\right] \operatorname{sen} \theta_{35}
$$

El área del plano B viene dada por:

$$
A_{b}=\frac{1}{2}\left[A C^{2} \frac{\operatorname{sen}^{2} \theta_{13} \operatorname{sen} \theta_{25}}{\operatorname{sen}^{2} \theta_{15} \operatorname{sen} \theta_{24}}-T C^{2} \frac{\operatorname{sen}^{2} \theta_{37} \operatorname{sen} \theta_{58}}{\operatorname{sen}^{2} \theta_{57} \operatorname{sen} \theta_{48}}\right] \operatorname{sen} \theta_{45}
$$

El área de la grieta de tracción viene dada por:

$$
A_{t}=\frac{1}{2}\left[T C^{2} \frac{\operatorname{sen} \theta_{34} \operatorname{sen} \theta_{35}}{\operatorname{sen} \theta_{49} \operatorname{sen} \theta_{57}}\right] \operatorname{sen} \theta_{79}
$$

Entonces el peso del bloque inestable de roca se puede expresar de la siguiente manera: 
$W=\frac{1}{6} \gamma n\left[A C^{3} \frac{\operatorname{sen}^{2} \theta_{13} \operatorname{sen} \theta_{25}}{\operatorname{sen}^{2} \theta_{15} \operatorname{sen} \theta_{24}}-T C^{3} \frac{\operatorname{sen}^{2} \theta_{37} \operatorname{sen} \theta_{58}}{\operatorname{sen}^{2} \theta_{57} \operatorname{sen} \theta_{48}}\right]$

donde

$$
\begin{aligned}
n= & \left(1-\cos ^{2} \theta_{34}-\cos ^{2} \theta_{35}-\cos ^{2} \theta_{45}+\right. \\
& \left.2 \cos \theta_{34} \cos \theta_{35} \cos \theta_{45}\right)^{\frac{1}{2}}
\end{aligned}
$$

Con respecto al cálculo de fuerzas debido a las presiones de agua $P$, para este desarrollo se ha considerado que el agua es libre de entrar al macizo rocoso a través de la grieta de tracción y las presiones de agua se consideran dependientes del gradiente hidráulico que se presenta a lo largo de las discontinuidades debido al flujo. En este análisis, se asumirá que el gradiente hidráulico varía de forma lineal a lo largo de las discontinuidades y que la máxima presión de agua se da en el punto $\mathrm{W}$.

$$
P=\gamma_{w} \frac{T C \operatorname{sen} \theta_{35}}{2 \operatorname{sen} \theta_{57}}+\left[\operatorname{sen} \phi_{7}+\frac{\operatorname{sen} \theta_{79}}{\operatorname{sen} \theta_{89}} \operatorname{sen} \phi_{7}\right]
$$

Es necesario aclarar que otras distribuciones de las presiones del agua diferentes a la considerada en el presente análisis pueden ocurrir, por lo tanto, queda a discreción del ingeniero, modificar las anteriores ecuaciones de manera que se acomoden mejor a las condiciones particulares que se estén analizando. La fuerza $V$ que actúa debido a la presión del agua en la grieta de tracción, es dependiente del volumen del triángulo de presiones que se forma en el área $\mathrm{A}_{\mathrm{T}}$ (cara de la grieta de tracción), por lo tanto, la presión en la grieta de tracción se puede expresar mediante la siguiente ecuación.

$$
V=\frac{1}{3} P A_{T}
$$

Asumiendo que todo el flujo de agua se transmite desde la grieta de tracción hacia los planos de discontinuidad A y B, la presión que actúa sobre dichos planos se podría expresar de la siguiente manera:

$$
\begin{aligned}
U_{a} & =\frac{1}{3} P A_{a} \\
U_{b} & =\frac{1}{3} P A_{b}
\end{aligned}
$$

Finalmente, es necesario obtener todas las fuerzas que actúan sobre la cuña inestable, el peso de la cuña $W$, reacción efectiva normal en el plano $\mathrm{A}, N_{\text {ae }}$, reacción

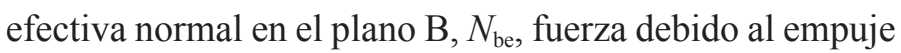
del agua en el plano $\mathrm{A}, U_{\mathrm{a}}$, fuerza debido al empuje del agua en el plano B, $U_{\mathrm{b}}$, fuerza debido al empuje del agua en la grieta de tracción $V$, y fuerza que actúa a lo largo de la línea potencial de deslizamiento $S$.

Con el fin de calcular las fuerzas que se oponen al deslizamiento de la cuña, se deben obtener las reacciones efectivas normales sobre los planos de deslizamiento A y $\mathrm{B}, N_{\text {ae }}$ y $N_{\text {be }}$, respectivamente.

$$
\begin{aligned}
& \left(N_{a e}+U_{a}\right)+m_{n a n b}\left(N_{b e}+U_{b}\right)+ \\
& m_{W n a} W+m_{V n a} V=0 \\
& \left(N_{b e}+U_{a}\right)+m_{n a n b}\left(N_{a e}+U_{a}\right)+ \\
& m_{W n b} W+m_{V n b} V=0
\end{aligned}
$$

Donde,

$$
\begin{aligned}
m_{n a n b}= & \operatorname{sen} \phi_{a} \operatorname{sen} \phi_{b} \cos \left(\alpha_{a}-\alpha_{b}\right)+ \\
& \cos \phi_{a} \cos \phi_{b} \\
m_{W n a}= & -\cos \phi_{a} \\
m_{W n b}= & -\cos \phi_{b} \\
m_{V n a}= & \operatorname{sen} \phi_{a} \operatorname{sen} \phi_{t} \cos \left(\alpha_{a}-\alpha_{t}\right)+\cos \phi_{a} \cos \phi_{t} \\
m_{V n b}= & \operatorname{sen} \phi_{b} \operatorname{sen} \phi_{t} \cos \left(\alpha_{b}-\alpha_{t}\right)+\cos \phi_{b} \cos \phi_{t}
\end{aligned}
$$

Resolviendo las ecuaciones anteriores se obtiene $N_{a e}$ y $N_{b e}$.

$N_{a e}=q W+r V-U_{a}$

$N_{b e}=x W+y V-U_{b}$

Donde:

$q=\frac{m_{n a n b} m_{W n b}-m_{W n a}}{1-m_{n a n b}^{2}}$

$r=\frac{m_{n a n b} m_{V n b}-m_{V n a}}{1-m_{n a n b}^{2}}$

$x=\frac{m_{n a n b} m_{W n a}-m_{W n b}}{1-m_{\text {nanb }}^{2}}$

$y=\frac{m_{n a n b} m_{V n a}-m_{V n b}}{1-m_{n a n b}^{2}}$

La fuerza desestabilizante $S$ que actúa a lo largo de la línea de intersección de los planos A y B, se resuelve mediante la siguiente expresión:

$S=m_{W 5} W+m_{V 5} V$

donde,

$m_{W 5}=\operatorname{sen} \phi_{5}$

$m_{V 5}=\cos \phi_{5} \operatorname{sen} \phi_{t} \cos \left(\alpha_{5}-\alpha_{t}\right)-\operatorname{sen} \phi_{5} \cos \phi_{t}$ 
El factor de seguridad para el caso estático se calcula como en (1), esto es, la razón entre las fuerzas resistentes y desestabilizantes, de donde resulta:

$$
F S=\frac{c_{a} A_{a}+c_{b} A_{b}+\left(q W+r V-U_{a}\right) \tan \phi_{a}+\left(x W+y V-U_{b}\right) \tan \phi_{b}}{m_{W 5} W+m_{V 5} V}
$$

\section{Análisis pseudo-estático}

El procedimiento de análisis pseudo-estático simula los movimientos del suelo como una fuerza estática que actúa desestabilizando el talud. La magnitud de esta fuerza está dada por el producto entre el peso del bloque deslizante $W$ y un coeficiente sísmico $k$ que actúa en dirección $\phi_{\mathrm{k}}$. El Factor de seguridad para el caso pseudo-estático teniendo en cuenta la fuerza sísmica se calcula de la siguiente manera:

$$
F S=\frac{c_{a} A_{a}+c_{b} A_{b}+\left(q(W-W k)+r V-U_{a}\right) \tan \phi_{a}+\left(x(W-W k)+y V-U_{b}\right) \tan \phi_{b}}{m_{W 5}(W+W k)+m_{V 5} V}
$$

\section{Análisis incluyendo la malla}

La Figura 4 muestra sombreado en color morado el área sobre la cual se aplica la fuerza distribuida $T$, distribuida sobre la cara $\mathrm{ABO}$ de la cuña inestable. Esta fuerza actúa como fuerza resistente en el sistema y presenta un ángulo de buzamiento $\phi_{\mathrm{T}} \mathrm{y}$ tiene una dirección de buzamiento $\alpha_{\mathrm{T}}$.

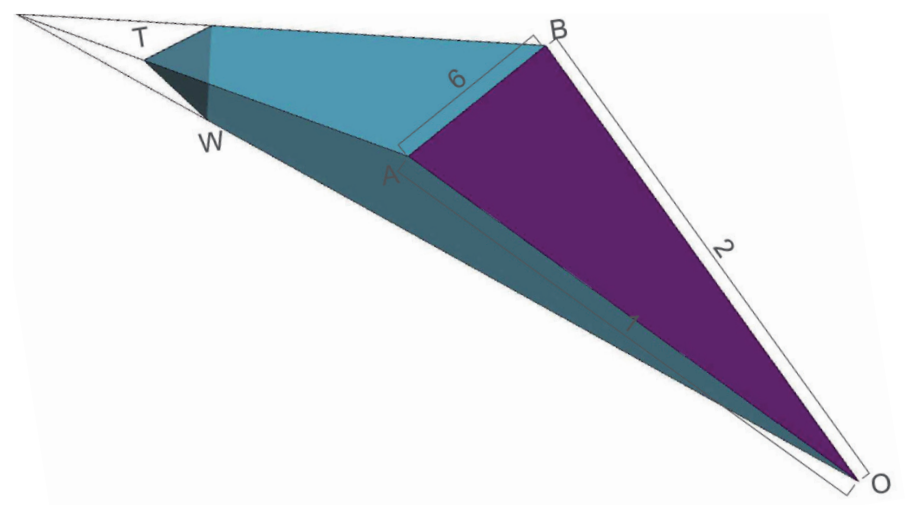

Figura 4: Geometría de la cuña de falla incluyendo el área de aplicación de la fuerza distribuida $T$ de la malla

Para resolver el problema se debe calcular el área superficial del talud sobre la cual actuaría la fuerza distribuida $T$. Inicialmente, se deben hallar los ángulos que se forman entre las líneas 1-6 y 2-6: $\theta_{16}=\arccos \left(\cos \phi_{1} \cos \phi_{6} \cos \left(\alpha_{1}-\alpha_{6}\right)+\operatorname{sen} \phi_{1} \operatorname{sen} \phi_{6}\right)$

$\theta_{26}=\arccos \left(\cos \phi_{2} \cos \phi_{6} \cos \left(\alpha_{2}-\alpha_{6}\right)+\operatorname{sen} \phi_{2} \operatorname{sen} \phi_{6}\right)$

Se debe considerar que la fuerza $T$ distribuida sobre el talud, tendrá un impacto diferente sobre el plano de deslizamiento A y el plano de deslizamiento B, por lo tanto, es necesario calcular el coeficiente $m$ de dicha fuerza para cada plano de discontinuidad, así como para la línea de intersección de los planos de la misma manera que se realizó anteriormente para las fuerzas debidas al peso del bloque y el empuje del agua.

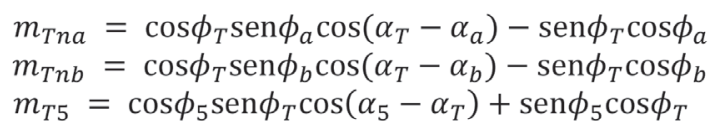

Finalmente se obtienen los coeficientes de la fuerza $T$ que actúa sobre cada uno de los planos A y B

$$
\begin{aligned}
& S=\frac{m_{n a n b} m_{T n b}-m_{T n a}}{1-m_{n a n b}^{2}} \\
& Z=\frac{m_{n a n b} m_{T n a}-m_{T n b}}{1-m_{n a n b}^{2}}
\end{aligned}
$$

Finalmente, se incluye en la ecuación planteada inicialmente para el cálculo del factor de seguridad estático la nueva fuerza actuante:

$F S=\frac{c_{a} A_{a}+c_{b} A_{b}+\left(q W+r V+s T A_{T}-U_{a}\right) \tan \phi_{a}+\left(x W+y V+z T A_{T}-U_{b}\right) \tan \phi_{b}}{m_{W 5} W+m_{V 5} V+m_{T 5} T A_{T}}$

Igualmente se debe tener en cuenta la nueva fuerza en el factor de seguridad pseudo-estático planteado inicialmente: $F S=\frac{c_{a} A_{a}+c_{b} A_{b}+\left(q(W-W k)+r V+s T A_{T}-U_{a}\right) \tan \phi_{a}+\left(x(W-W k)+y V+z T A_{T}-U_{b}\right) \tan \phi_{b}}{m_{W 5}(W+W k)+m_{V 5} V+m_{T 5} T A_{T}}$

\section{Falla por volcamiento}

\section{Análisis estático}

Con el fin de hallar el factor de seguridad en condición estática, es necesario determinar los momentos que ejercen las fuerzas involucradas en el sistema (peso del bloque y presión del agua). Para el siguiente desarrollo se calcularán los momentos con respecto al punto O:

$$
W=\left(h b+0.5 b^{2} \tan \alpha\right) s \gamma
$$

donde $s$ es el ancho del bloque. Se considera que el efecto de la presión del agua en el contorno del bloque deslizante es función del gradiente hidráulico que exista entre los 
puntos $\mathrm{OA}$ y $\mathrm{CB}$, asumiendo que en los puntos $\mathrm{O}$ y B la presión del agua es 0 . Se asume que el gradiente hidráulico varía de forma lineal a lo largo del contorno del bloque como se muestra en la Figura 5.

$$
\begin{aligned}
& P_{A}=\frac{1}{6} h_{b} \gamma_{w} h^{2} s \\
& P_{B}=\frac{1}{6}\left(h_{2}+H\right)^{2} h_{a} s \gamma_{w}
\end{aligned}
$$

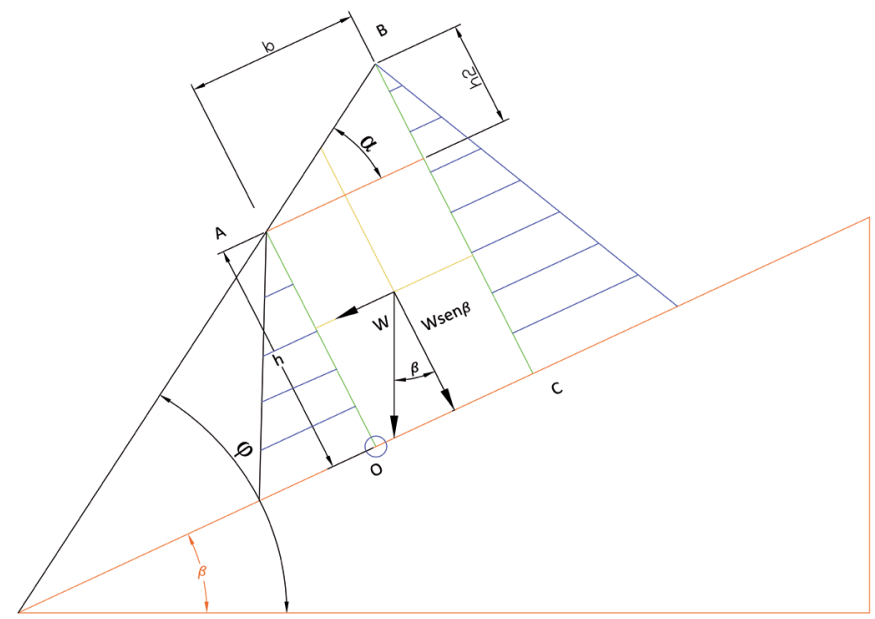

Figura 5: Fuerzas involucradas en el análisis para falla en cuña

Cálculo de los momentos estabilizantes:

$$
M_{E}=W \cos \beta \frac{b}{2}+\frac{1}{6} h_{b} \gamma_{w} h^{2} s
$$

Cálculo de los momentos desestabilizantes:

$$
M_{D}=W \operatorname{sen} \beta\left(\frac{h}{2}+\frac{1}{3} h_{2}\right)+\frac{1}{6}\left(h_{2}+H\right)^{2} h_{a} s \gamma_{w}
$$

Cálculo del factor de seguridad:

$$
F S=\frac{W \cos \beta \frac{b}{2}+\frac{1}{6} h_{b} \gamma_{w} h^{2} s}{W \operatorname{sen} \beta\left(\frac{h}{2}+\frac{1}{3} h_{2}\right)+\frac{1}{6}\left(h_{2}+H\right)^{2} h_{a} s \gamma_{w}}
$$

\section{Análisis pseudo-estático}

El procedimiento de análisis pseudo-estático simula los movimientos del suelo como una fuerza estática que actúa desestabilizando el talud. La magnitud de esta fuerza está dada por el producto entre el peso del bloque deslizante $W$ y un coeficiente sísmico $k$ que actúa en dirección $\phi_{\mathrm{k}}$ El Factor de seguridad para el caso pseudo-estático teniendo en cuenta la fuerza sísmica, se calcula de la siguiente manera:

$$
F S=\frac{W \cos \beta \frac{b}{2}+\frac{1}{6} h_{b} \gamma_{w} h^{2} s}{W \operatorname{sen} \beta\left(\frac{h}{2}+\frac{1}{3} h_{2}\right)+\frac{1}{6}\left(h_{2}+H\right)^{2} h_{a} s \gamma_{W}+W \cos \left(\beta+\phi_{k}\right) k \frac{b}{2}+W k \operatorname{sen}\left(\beta+\phi_{k}\right)\left(\frac{h}{2}+\frac{1}{3} h_{2}\right)}
$$

\section{Análisis incluyendo la malla}

Con el fin de incluir el efecto estabilizante que una malla metálica pueda tener al momento del cálculo del factor de seguridad, se considera en los momentos estabilizantes el efecto de una fuerza distribuida $P$ sobre la superficie del talud inestable como se muestra en la Figura 6. El caso estático y pseudo-estático vienen dados por:

$$
\begin{aligned}
& F S=\frac{W \cos \beta \frac{b}{2}+\frac{1}{6} h_{b} \gamma_{w} h^{2} s+\frac{1}{2} P s b \cos \alpha+\left(h+\frac{1}{3} h 2\right) P \operatorname{sisen} \alpha}{W \operatorname{sen} \beta\left(\frac{h}{2}+\frac{1}{3} h_{2}\right)+\frac{1}{6}\left(h_{2}+H\right)^{2} h_{a} s \gamma_{w}}(44 \mathrm{a}) \\
& F S=\frac{W \cos \beta \frac{b}{2}+\frac{1}{6} h_{b} \gamma_{w} h^{2} s+P s b \cos \alpha \frac{b}{2}+P s b \operatorname{sen} \alpha\left(h+\frac{1}{3} h_{2}\right)}{W \operatorname{sen} \beta\left(\frac{h}{2}+\frac{1}{3} h_{2}\right)+\frac{1}{6}\left(h_{2}+H\right)^{2} h_{a} s \gamma_{w}+W \cos \left(\beta+\phi_{k}\right) K_{t} \frac{b}{2}+W K_{t} \operatorname{sen}\left(\beta+\phi_{k}\right)\left(\frac{h}{2}+\frac{1}{3} h_{2}\right)}
\end{aligned}
$$

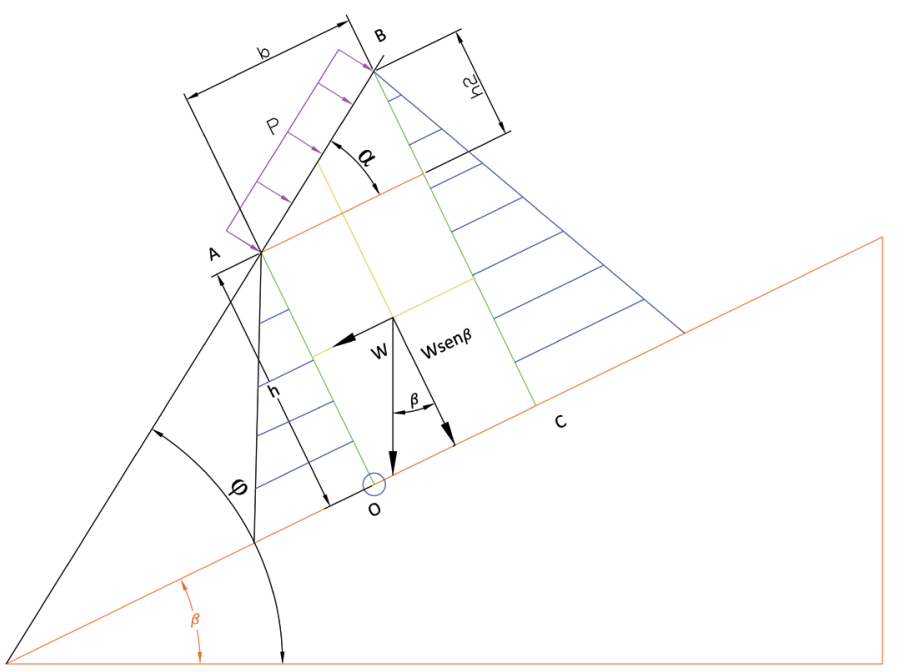

Figura 6: Fuerzas involucradas en el análisis para falla en cuña

\section{Metodología para el cálculo de estabilizaciones con mallas metálicas flexibles}

Castro (2000) realizó estudios sobre los modelos de funcionamiento de los sistemas flexibles como elemento de soporte para la estabilización de taludes. Estos estudios sirvieron como insumo para el desarrollo de la metodología de diseño de mallas metálicas flexibles que se presenta a continuación. La malla se considera como un material continuo, aunque en la realidad no lo es. Dado que la malla carece de resistencia a la flexión, no se considera su espesor y los parámetros de la misma se emplearán siempre por unidades de longitud y no de superficie, en lo que a su sección se refiere. La Figura 7 muestra un esquema de la malla. 


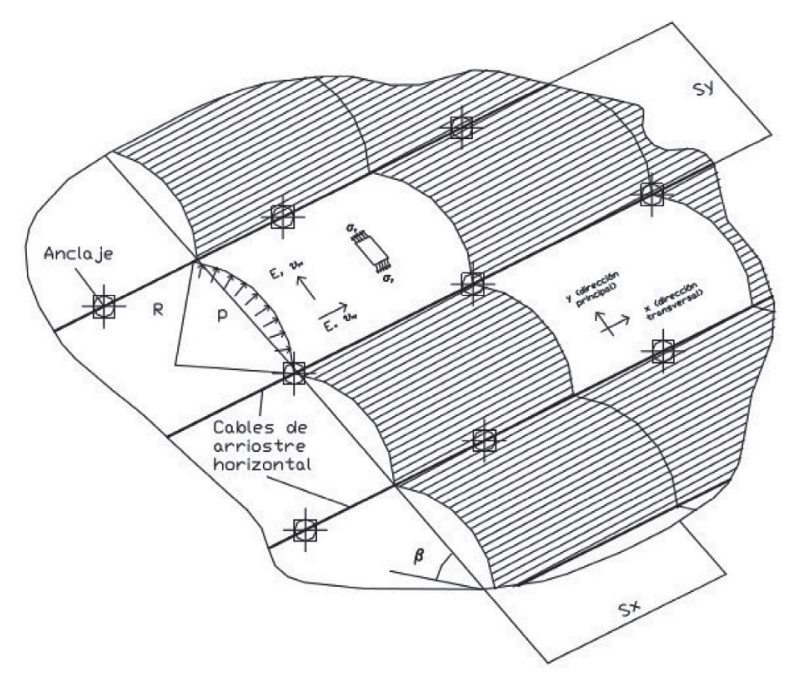

Figura 7: Esquema del modelo unidireccional (Castro, 2000)

De acuerdo a las características geométricas de la deformada mostrada en la Figura 8, se tiene que la deformación transversal de la malla es nula y compatibilizando con los parámetros mecánicos de la membrana se obtiene la siguiente expresión que representa el desplazamiento vertical en función de la presión que aplica la malla al talud.

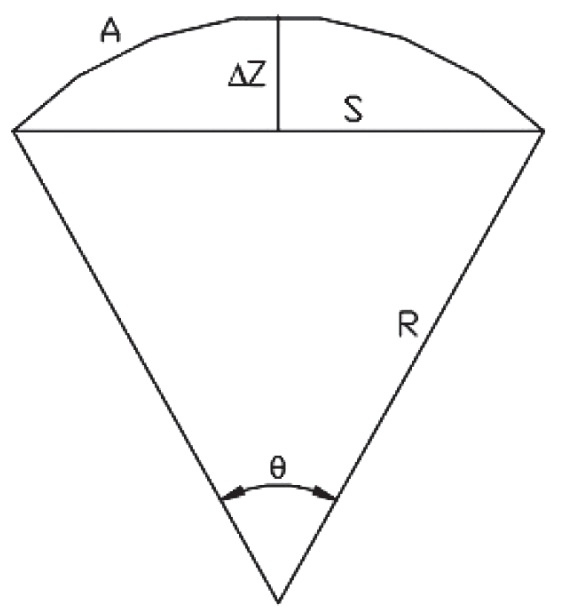

Figura 8: Esquema de la deformada de la malla (Castro, 2000)

$$
\begin{aligned}
& \frac{\theta}{2}=180-2 \arctan \left(\frac{S y}{2 \Delta Z}\right) \\
& R=\frac{S}{2 \operatorname{sen} \frac{\theta}{2}} \\
& A=R \theta \\
& \Delta Z=f=\left(\frac{S^{2}\left(\frac{24 p S}{E_{y c}}\right)^{\frac{2}{3}}}{64-\left(\frac{96 p S}{E_{y c}}\right)^{\frac{2}{3}}}\right)^{0.5}
\end{aligned}
$$

Donde $p$ es la presión unitaria en $\mathrm{kN} / \mathrm{m}^{2}, S$ es la separación entre arriostres en $\mathrm{m}, E_{\mathrm{yc}}$ es el módulo de elasticidad longitudinal en la dirección $Y$ en $\mathrm{kN} / \mathrm{m}, \Delta Z$ es el desplazamiento vertical del punto central o flecha en $\mathrm{mm}$, $\theta$ es el ángulo central, $R$ es el radio de curvatura y $A$ es la longitud del arco.

Por otro lado, Blanco-Fernandez et al. (2011), utilizaron una relación entre la fuerza que ejerce la tensión que se presenta, la longitud del cable con curvatura y la flecha, partiendo de las consideraciones de asumir un contacto con forma parabólica con una longitud y una flecha definida, y considerando que la curvatura del cable adopta una forma parabólica, los valores de fuerza por cada cable al terreno vendrían definidos por la expresión presentada a continuación:

$$
F=p L \approx \frac{8 T}{\sqrt{\frac{L^{2}}{f^{2}}+16}}
$$

donde $F$ es la fuerza total aplicada, $p$ es la fuerza por unidad de longitud, $L$ es la longitud del cable con curvatura, $T$ es la fuerza de tracción en el cable y $f$ es la flecha en el centro de la luz. La ecuación (46) puede reorganizarse y presentarse de la siguiente forma.

$$
T=\frac{p L \sqrt{\frac{L^{2}}{f^{2}}+16}}{8}
$$

La membrana es activa con la apropiada fuerza de pretensión del sistema. Si la membrana se instala con una fuerza $T$ de pretensión conocida y controlada y la superficie del talud presenta una forma convexa parabólica con una flecha en el centro luz de cada paño $f$, entonces es posible admitir que la membrana esté ejerciendo una fuerza inicial $p$ sobre la superficie del talud. Igualmente, Blanco-Fernandez et al. (2011) encontraron una relación entre la fuerza en el anclaje en función de la separación en $Y$, rigidez de la malla y tensión en la membrana. Cabe resaltar que la expresión fue obtenida producto de las modelaciones de taludes en suelo con la herramienta computacional ANSYS, como se presentan a continuación.

$$
F_{\text {perno }}=(-2.81+4.06 S y-0.0271 K+0,114 T)^{2}
$$

Donde $S y$ es la separación de los pernos, $K$ es el módulo de compresibilidad de la malla, $T$ es la tensión en la malla y $F_{\text {perno }}$ es la fuerza axial en el perno. Del remplazo de las ecuaciones (46) y (47) en (48), se puede obtener una 
expresión de la forma:

$$
F_{\text {perno }}=f(S y, K, P)
$$

Finalmente, se modeló (49) obteniendo las gráficas de la presión en la malla de acuerdo a:

$$
P=f\left(S y, K, F_{\text {perno }}\right)
$$

\section{Resultados y análisis}

A continuación, se presentan los resultados y su respectivo análisis tanto para las mallas, el macizo rocoso en diferentes procesos de inestabilidad y el sistema de macizo rocoso estabilizado con sistema flexible.

\section{Falla planar}

Los resultados de falla planar en condición estática sin malla metálica flexible fueron realizados partiendo de escenarios de ángulo del talud de $\beta=45,60,75$ y $90^{\circ}$, así como buzamiento de las discontinuidades constante de $\alpha=22.5^{\circ}$. Es de notar que en las modelaciones se han considerado como constantes los siguientes parámetros: $\gamma=$ $26 \mathrm{kN} / \mathrm{m}^{3}$, distancia horizontal $b=0.5 \mathrm{~m}$, altura de la grieta de tracción $z=0.25 \mathrm{~m}$ y grieta de tracción completamente llena de agua. Las Figuras 9 a 12 muestran resultados de la variación del factor de seguridad FS en función del número de estabilidad $c /(\gamma H)$ para diferentes valores del ángulo de inclinación del talud.

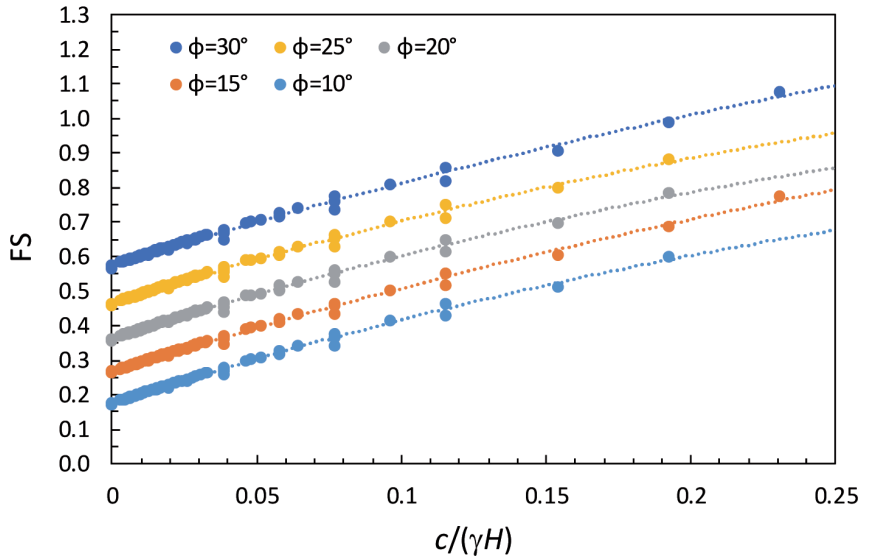

Figura 9: Variación del factor de seguridad FS para $\beta=45^{\circ}$ (falla planar en condición estática)

En general se aprecia que el comportamiento para diferentes ángulos de fricción es similar y los factores de seguridad aumentan a medida que aumenta el número de estabilidad. Del mismo modo FS también aumenta cuando aumenta el ángulo de fricción. Por lo tanto, es notorio que las gráficas pierden pendiente a medida que aumenta la inclinación del talud, comportamiento que se aprecia claramente en la Figura 13 y que se repite para diferentes números de estabilidad.

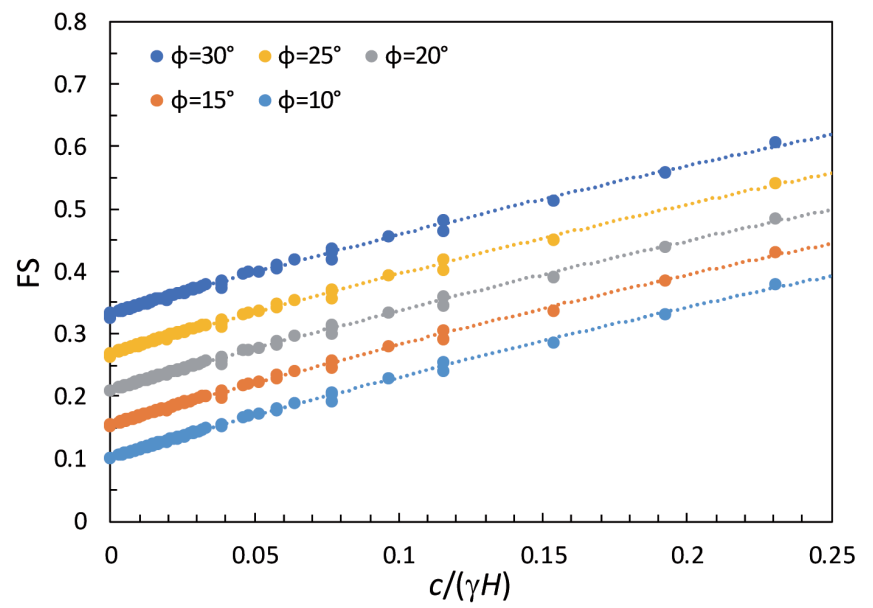

Figura 10: Variación factor de seguridad FS para $\beta=60^{\circ}$ (falla planar en condición estática)

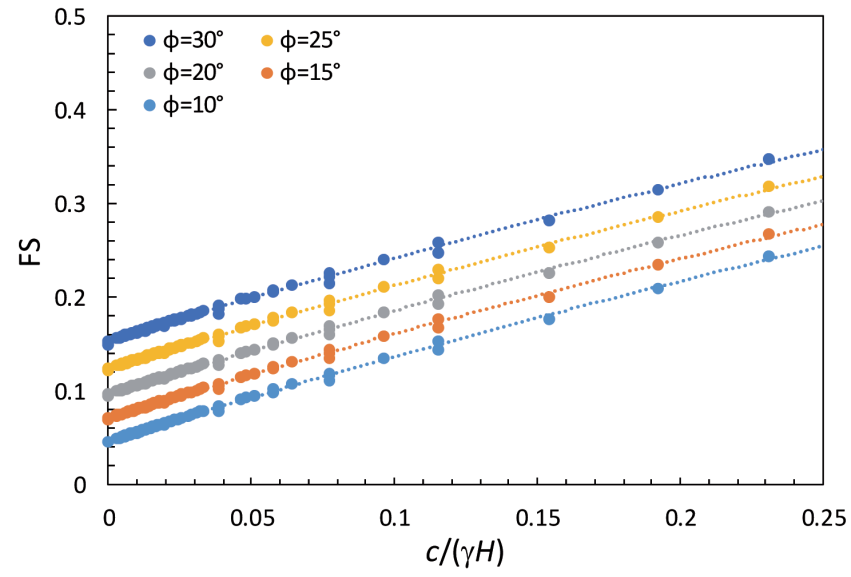

Figura 11: Variación factor de seguridad FS para $\beta=75^{\circ}$ (falla planar en condición estática)

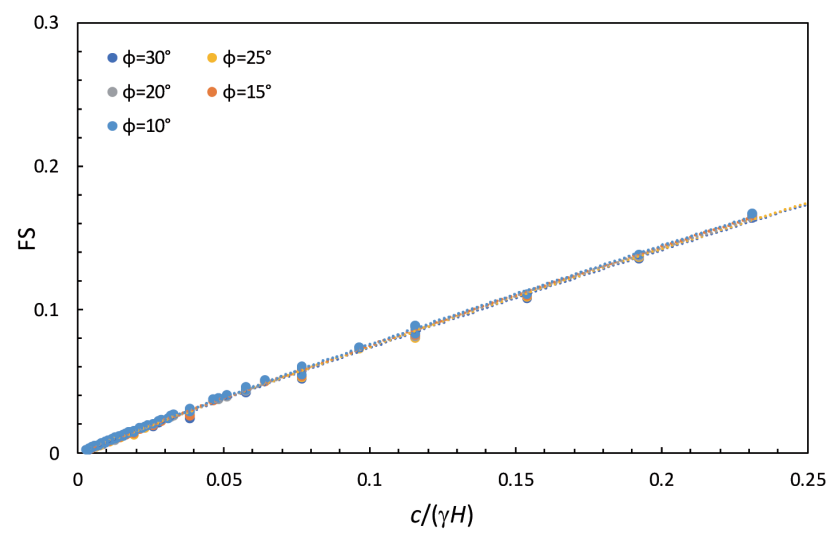

Figura 12: Variación factor de seguridad FS para $\beta=90^{\circ}$ (falla planar en condición estática) 


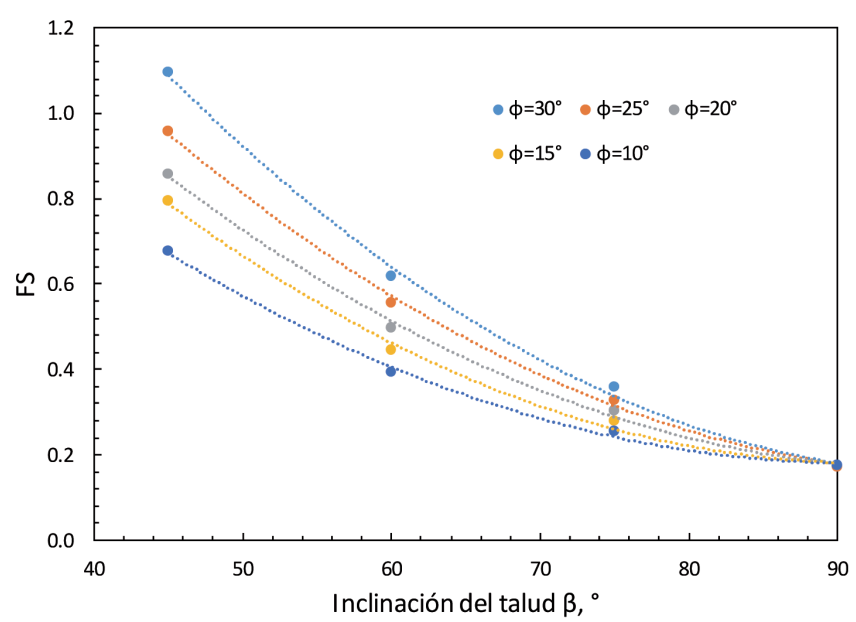

Figura 13: Variación factor de seguridad FS en función de $\beta$ para $c /(\gamma H)=0.25$ (falla planar en condición estática)

El comportamiento mostrado en la Figura 13 se mantiene en condición pseudo-estática sin la implementación del sistema de contención con malla metálica flexible. Sólo que el factor de seguridad se hace menor en las mismas condiciones. Por otro lado, luego de la implementación del sistema de contención con malla, se obtienen los resultados en condición estática y macizo rocoso completamente saturado, tal como se muestra en la Figura 14.

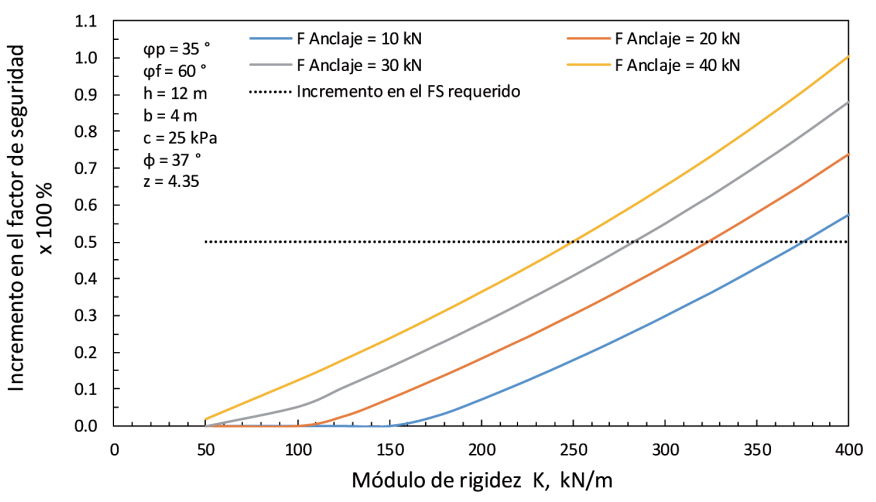

Figura 14: Incremento en el factor de seguridad estático FS (x $100 \%$ ) versus el módulo de rigidez de la malla (falla planar)

La Figura 14 muestra los resultados de los cálculos realizados para el sistema talud inestable más malla estabilizadora para diferentes condiciones de análisis. La línea negra punteada representa en todas las gracias el incremento en el factor de seguridad que es requerido para cumplir con la normativa de diseño colombiana. Se pueden observar los incrementos en los factores de seguridad obtenido gracias a la acción estabilizadora que ejerce la malla metálica versus la variación en el módulo de rigidez de la malla para diferentes fuerzas de anclaje.
En la Figura 15 se puede ver claramente la influencia de la rigidez y la fuerza de los anclajes en el aporte a la estabilidad del talud. Analizando esta gráfica y siguiendo inicialmente la línea roja punteada, podremos observar que, para una misma fuerza de anclaje, el incremento del factor de seguridad puede variar de un $0 \%$ a un $80 \%$ si se aumenta la rigidez de la malla desde $50 \mathrm{kN} / \mathrm{m}$ a $400 \mathrm{kN} / \mathrm{m}$.

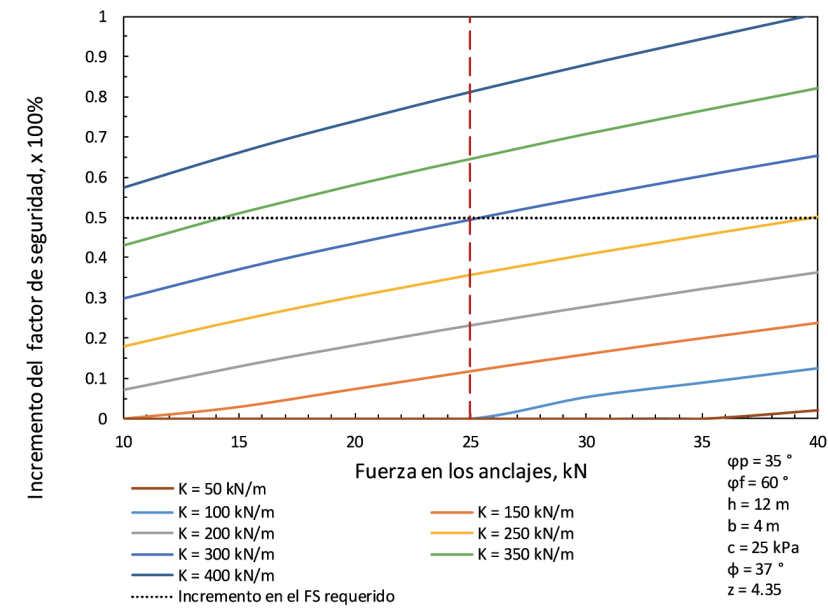

Figura 15: Incremento en el factor de seguridad estático FS (x $100 \%$ ) versus la fuerza en los anclajes (falla planar)

\section{Falla cuña}

Para los siguientes análisis de falla en cuña, se ha emulado un número de estabilidad, con el fin de incluir la mayor parte de parámetros de resistencia, y con esto saber cuál es la influencia de estos en la estabilidad de los taludes. El número de estabilidad es $c /(\gamma \mathrm{AO})$, donde $\mathrm{AO}$ es una longitud que está relacionada con el tamaño del bloque. Mayores valores de AO implican mayores tamaños del bloque. La Figura 16 presenta los resultados de FS versus $c /(\gamma \mathrm{AO})$ variando el ángulo de fricción, obtenidos para la modelación de falla en cuña.

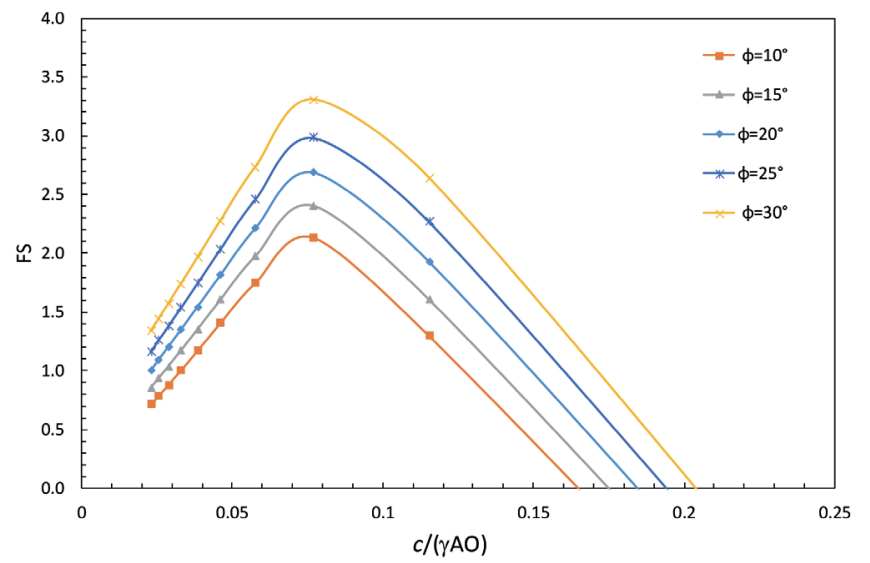

Figura 16: Variación del factor de seguridad FS en función de AO (falla en cuña en condición estática) 
En la Figura 16 se puede observar un comportamiento marcado en estos tipos de fallas, es así como, se genera un máximo de resistencia para valores de número de estabilidad alrededor de 0.075. A partir de $c /(\gamma \mathrm{AO})$ de 0.1 el factor de seguridad cae dramáticamente. El comportamiento para diferentes ángulos de fricción tiende a ser el mismo. De este análisis se tiene que el comportamiento está gobernado por el tamaño del bloque, es decir, se genera un tamaño que hace máximo el factor de seguridad. Este resultado es muy importante, pues cuando se quiera estabilizar con maya metálica flexible se debe apuntar a enfocarse en los tamaños que maximizan el factor de seguridad en el bloque. El comportamiento es similar para la condición pseudoestática, con una disminución del factor de seguridad.

A continuación, se presentan los resultados de la condición de procesos de inestabilidad con solución de malla metálica flexible para el caso estático considerando un macizo rocoso completamente saturado. En las Figuras 17, 18 y 19, se muestran los resultados de los cálculos realizados para el sistema talud inestable más malla estabilizadora para diferentes condiciones de análisis, la línea negra punteada representa el incremento en el factor de seguridad que es requerido para cumplir con la normativa de diseño colombiana.

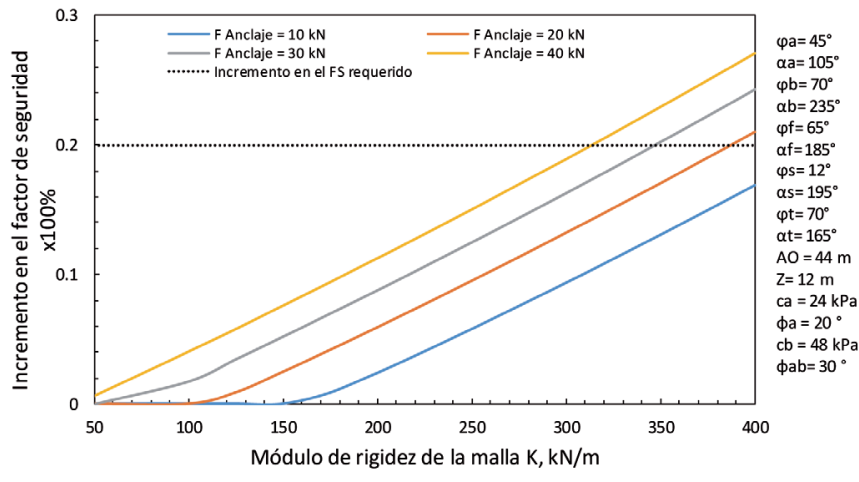

Figura 17: Incremento en el factor de seguridad estático FS (x $100 \%$ ) versus el módulo de rigidez de la malla (falla en cuña)

Teniendo en cuenta la línea que indica el incremento esperado en el factor de seguridad, podemos observar que existen diferentes combinaciones de rigidez y separación en los anclajes con las cuales es posible obtener los factores de seguridad deseados, por lo tanto, es posible encontrar una combinación con la cual se puedan optimizar los costos de la construcción del sistema de estabilización.

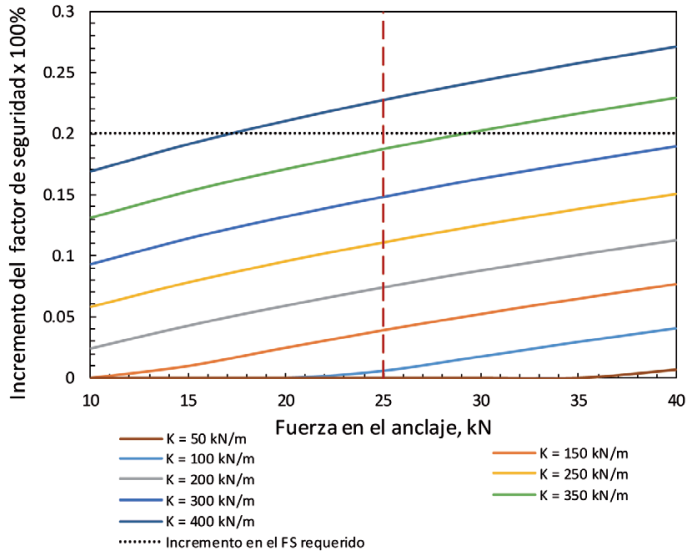

Figura 18: Incremento en el factor de seguridad estático FS (x $100 \%$ ) versus la fuerza en el anclaje (falla en cuña)

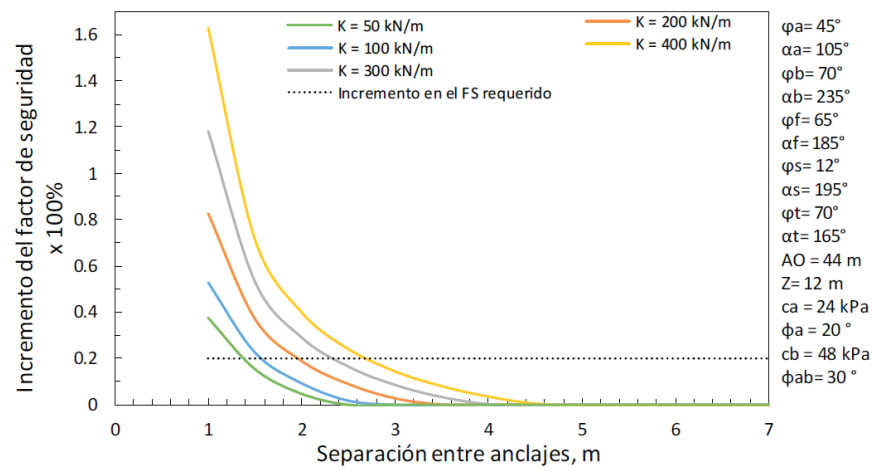

Figura 19: Incremento en el factor de seguridad estático FS (x $100 \%$ ) versus la separación de los anclajes (falla en cuña)

\section{Falla volcamiento}

Para la falla en volcamiento se ha elaborado la gráfica mostrada en la Figura 20 que relaciona el factor de seguridad con el ángulo de talud, para diferentes relaciones de ancho y altura $b / h$, debido a que la falla en general depende de este parámetro. No ha sido necesario realizar normalización de esta variable independiente

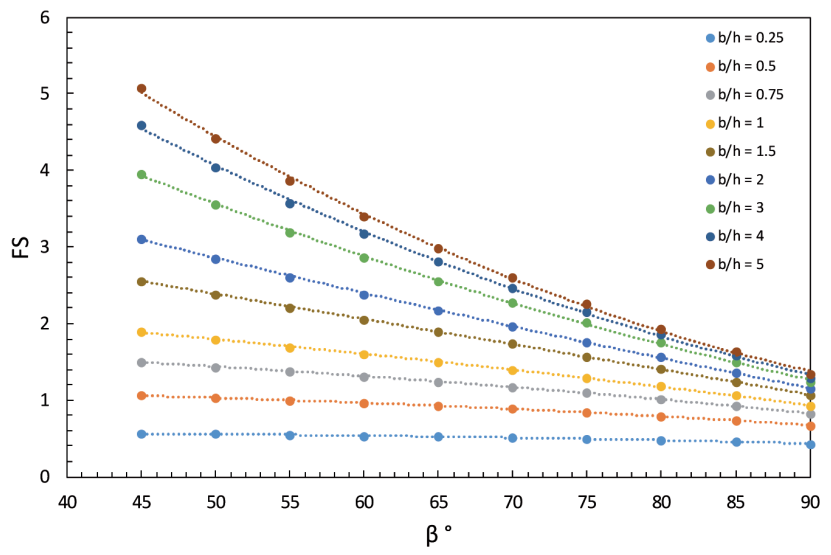

Figura 20: Variación del factor de seguridad FS en función de la inclinación del talud y $b / h$ (falla en volcamiento en análisis estático) 
Se puede apreciar en la Figura 20 que, relaciones de $b / h$ mayores, generan mayores factores de seguridad, pero FS tiende a disminuir a medida que aumenta el ángulo del talud. Así mismo relaciones de $b / h$ menores que 0.5 , prácticamente tiende a volcarse, por lo que el elemento tiende a ser inestable por sí solo. Algo importante en esta figura es que a medida que aumenta la inclinación de talud, el valor tiende a ser el mismo, pasando de diferencias de varias unidades de factor de seguridad a casi una sola. Por lo que el ángulo del talud es el factor de mayor influencia en inclinaciones medias y bajas (menores a $60^{\circ}$ ) la relación $b / h$ tiende a no ser importe en ángulos mayores a $80^{\circ}$, en contraste con la gran importancia de este parámetro para inclinaciones bajas.

Las Figuras 21, 22 y 23 presentan las gráficas correspondientes al análisis de volcamiento con la solución de malla metálica flexible.

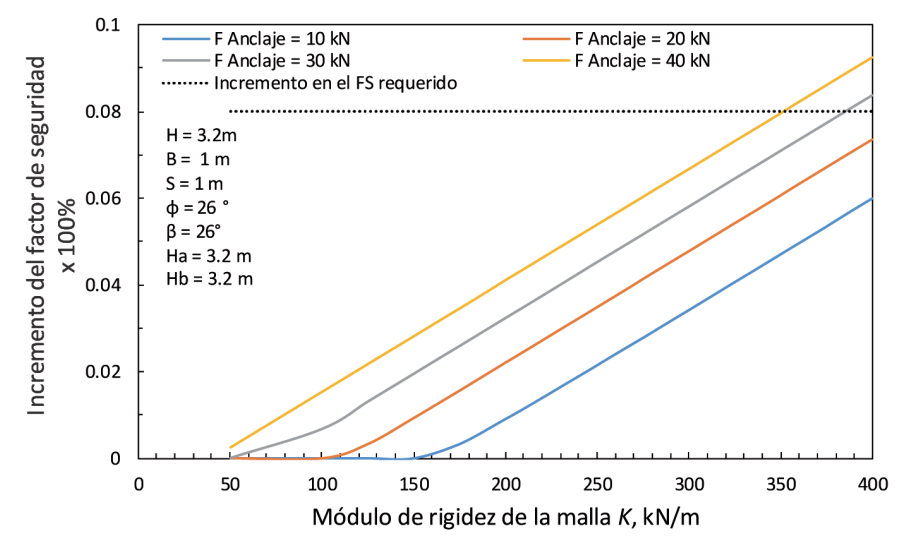

Figura 21: Incremento en el factor de seguridad estático FS (x $100 \%$ ) versus el módulo de rigidez de la malla (falla por volcamiento)

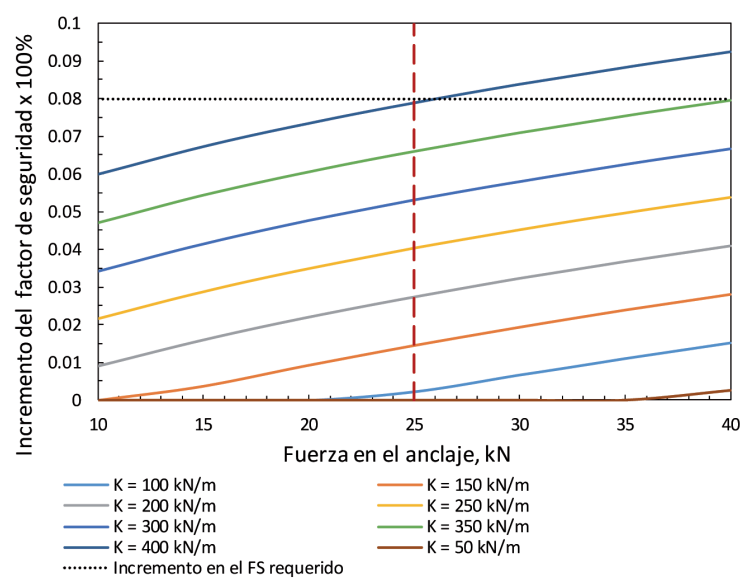

Figura 22: Incremento en el factor de seguridad estático FS (x $100 \%$ ) versus la fuerza en el anclaje (falla por volcamiento)

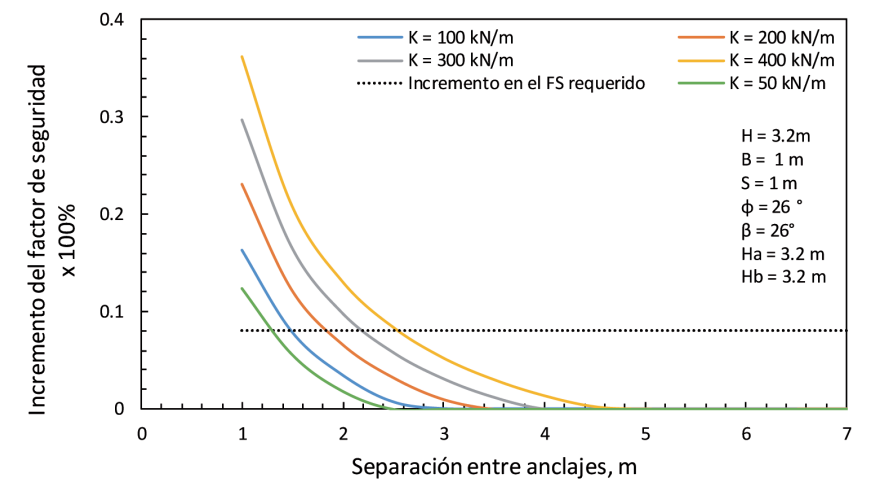

Figura 23: Incremento en el factor de seguridad estático FS (x $100 \%$ ) versus la separación de los anclajes (falla por volcamiento)

\section{Discusión}

Los sistemas flexibles de alta resistencia son uno de los muchos sistemas de estabilización existentes para taludes en roca. Estos están compuestos por una malla metálica de alta resistencia y una red de cables de refuerzo y anclajes, que en conjunto logran aportar una presión estabilizadora sobre la superficie de un talud. El método para el diseño de soluciones flexibles en la estabilización de taludes con sistema malla metálica flexible y anclajes desarrollado en la presente investigación, se basa en asumir que la membrana presenta un comportamiento activo, que debe ejercer una presión de diseño sobre el macizo rocoso desde el momento de su instalación y que la forma del terreno es cóncava. Teniendo en cuenta lo anterior, solo se podrá lograr resultados satisfactorios para un talud real, si es posible cumplir con las condiciones de diseño establecidas en las hipótesis de cálculo. Por ejemplo, se debe verificar que no exista punzonamiento sobre las mallas. Se propone el uso de anclajes activos para tensionar la malla metálica y de esta manera conseguir que el sistema trabaje de manera activa en vez de los pernos roscados que tradicionalmente se utilizan en este tipo de instalaciones. Se presume que la utilización de estos pernos y no de anclajes de tensionamiento en la instalación de estos sistemas no permite que se desarrolle en la malla metálica la tensión suficiente que permita que esta se trasmita al macizo rocoso y de esta manera que el sistema trabaje de forma activa. Teniendo en cuenta lo anterior se hace necesario continuar con futuras investigaciones realizando ensayos de campo a escala real con el fin de instrumentar este sistema basando los diseños en la metodología planteada en el presente 
trabajo con el fin de verificar su funcionamiento y definir cuál sería la forma apropiada de instalación del sistema para lograr el comportamiento activo del cual se parte como hipótesis de diseño.

En general se puede observar que el parámetro de mayor influencia en el diseño de las mallas metálicas es el módulo de rigidez de la malla, debido a que los aumentos progresivos en este parámetro consiguen los mayores aumentos en el factor de seguridad, sin embargo, el efecto de estos aumentos varía notablemente dependiendo del tipo de falla que se esté analizando, así como de las condiciones geométricas y geomecánicas del macizo rocoso que se esté evaluando. Con respecto a la separación de los anclajes, se puede observar que, para separaciones de anclajes muy bajas, se puede alcanzar el incremento en el factor de seguridad esperado para los tres casos de falla analizados aun cuando las rigideces de la malla son pequeñas. Igualmente, se puede establecer que existen diferentes combinaciones de rigideces de la malla, fuerza de anclaje y separación de los mismos, con las cuales se podría estabilizar el talud, por lo tanto, es posible realizar optimizaciones al diseño del sistema que permitan reducir costos y tiempos de construcción. Se puede observar que la separación en los anclajes de 3 x $3 \mathrm{~m}$ que se usa en la construcción de este tipo de estabilizaciones de manera frecuente, no funciona para el caso específico de estudio, es decir, la decisión del diseñador de la separación que se debe usar para realizar la estabilización debe ser tomada teniendo en cuenta las condiciones geométricas y geomecánicas del talud específico de estudio.

Los resultados permiten explicar el por qué del bajo nivel de funcionamiento que presenta este tipo de estabilizaciones en el contexto colombiano. Generalmente estos sistemas no funcionan como estabilizaciones de tipo activo a pesar de que la hipótesis de diseño de las mismas asume esta condición, si no funcionan como elementos de contención ante las caídas de bloques de roca o flujo de detritos en los taludes. Esto se debe principalmente a dos cosas: las mallas metálicas que se utilizan generalmente para la construcción de estos sistemas, no son mallas de altos módulos de rigidez diseñadas para estos fines, si no corresponden a mallas de tipo cerramiento de triple o doble torsión. Teniendo en cuenta la gran influencia que tiene la rigidez en el aumento del factor de seguridad y con los análisis realizados para mallas metálicas de bajo módulo, se puede observar el aporte casi insignificante que una malla de estas condiciones hace a la estabilidad general del talud. Por otro lado, los diseños realizados para este tipo de estabilizaciones no tienen en cuenta que, para mallas metálicas de muy bajos módulos de rigidez, es necesario utilizar separaciones en los anclajes muy bajas (menores a $1.5 \mathrm{~m}$ en el mejor de los casos) para alcanzar el aumento en el factor de seguridad deseado. Por lo general se utilizan separaciones que se encuentran entre los 2 × 2 y 3 × $3 \mathrm{~m}$ con los cuales se pudo evidenciar que para ninguno de los casos analizados, las mallas de bajo módulo de rigidez no realizan aporte alguno a la estabilidad del talud. La efectividad de estos sistemas de contención, se encuentra limitada por las separaciones en los anclajes, se estableció que para separaciones mayores a $4 \times 4 \mathrm{~m}$, ninguna malla, inclusive las de alto módulo de rigidez presenta aporte a la estabilidad del talud.

Entendiendo que los resultados mostrados en los gráficos corresponden a casos de análisis para macizos rocosos específicos, estos solo pueden ser usados para evaluar la influencia en general de los parámetros de entrada en el diseño mas no como ábacos de diseño para cualquier tipo de talud.

\section{Conclusiones}

A partir de los resultados presentados y analizados y discutidos, se pueden derivar las siguientes conclusiones.

El incremento del factor de seguridad en taludes en falla planar está mayormente influenciada por la variación de la cohesión. Esta propiedad, junto con la altura del talud son los únicos factores influyentes para ángulos de inclinación cercanos a los $90^{\circ}$, por lo cual el sistema en este escenario no trabaja, pues su aporte en el factor de seguridad es sobre la fricción en la discontinuidad. El factor de seguridad para falla en cuña está influenciado mayoritariamente por el tamaño del bloque. Existe un número de estabilidad $c /(\gamma H)$ que hace máximo el factor de seguridad para cada caso en particular, después el factor de seguridad decrece hasta la falla. El módulo de rigidez de la malla es el parámetro de mayor influencia en el aporte al factor de seguridad para las estabilizaciones con sistema malla metálica flexible más anclajes.

Se estableció que estabilizaciones con separaciones en 
los anclajes mayores a $4 \times 4 \mathrm{~m}$, no tienen aporte en la estabilidad del talud, pues el sistema una vez que alcanza esta separación pasa de funcionar con una condición activa a una condición pasiva. Para lograr aumentos en el factor de seguridad con los cuales se cumpla la normativa colombiana utilizando mallas de bajo módulo de rigidez, es necesario utilizar separaciones en los anclajes muy bajas ( $<1.5$ o 1 m según el caso de estudio).

Los sistemas de estabilización flexible con mallas no funcionan por lo general en el contexto colombiano, debido a que es usual utilizar mallas de bajas resistencia (tipo cerramiento de triple o doble torsión), con separaciones de anclajes que son usadas frecuentemente en sistemas constructivos ( 2 × 2 y 3 × 3 m). Esta combinación de bajo módulo de rigidez-separación de anclajes alta, implica que el aporte a la estabilidad del sistema es casi nulo, por lo tanto, el sistema pasa de trabajar en condición activa a condición pasiva como elemento de retención.

Con el método de cálculo que se ha presentado y discutido en esta investigación, es posible obtener soluciones de ingeniería a los problemas de inestabilidades en macizos rocosos, teniendo en cuenta factores como la rigidez de la malla, fuerza en los anclajes, y las propiedades geomecánicas y geométricas de los taludes.

\section{Referencias}

Blanco-Fernandez, E., Castro-Fresno, D., Díaz, J.D.C. and Lopez-Quijada, L. (2011). Flexible systems anchored to the ground for slope stabilisation: Critical review of existing design methods. Engineering Geology 122(3), 129-145

Caballero Chaves, O.J. (2011). Base de datos de deslizamientos inducidos por sismos. Tesis de pregrado, Universidad Javeriana Cała, M., Flum, D., Roduner, A., Rüegger, R. and Wartmann, S. (2012). TECCO $®$ slope stabilization system and RUVOLUM $®$ dimensioning method. AGH University of Science and Technology, Faculty of Mining and Geoengineering, Romanshorn, Switzerland
Cardoso, A.I.S. (2005). Estabilização de taludes rochosos com redes metálicas pré-tensionadas (Rock slope stabilization with pretensioned metalic meshes). Master's thesis, Coimbra University, Portugal (en portugués)

Castro, D. (2000). Estudio y análisis de las membranas flexibles como elemento de soporte para la estabilización de taludes y laderas de suelos y/o materiales sueltos. Tesis doctoral, Universidad de Cantabria, España

Fariñas de Alba, J.L., Gómez Prieto, R., Mataix González, C., García Bermudez, P., Llopis Trillo, G., Serrano Pedraza, P.A. y López Jimeno, C. (2008). Manual de estabilización y revegetación de taludes. ETSI Minas y Energía UPM, Madrid

Hoek, E. and Bray, J.D. (2014). Rock slope engineering. Third edition, CRC Press

Peila, D., Oggeri, C. and Castiglia, C. (2007). Ground reinforced embankments for rockfall protection: design and evaluation of full scale tests. Landslides 4(3), 255-265

Ramos, A.M., Trujillo-Vela, M.G. y Prada, L.F. (2015). Análisis descriptivos de procesos de remoción en masa en Bogotá. Obras y Proyectos 18, 63-75

Salazar Hernández, J.D. (2012). Zonificación de amenaza por deslizamientos inducidos por sismo. Tesis de pregrado, Universidad Javeriana 